\title{
The journey to setting up a comprehensive gynecologic cancer service in Upper Egypt
}

\author{
Ahmed Abdelbadee $\mathbb{~ ( 1 ) ~ , ~ A s s i u t , ~ E g y p t ~ a n d ~ H i s h a m ~ A b o u - T a l e b , ~ A s s i u t , ~ E g y p t ~}$
}

Egypt is a lower-middle income country located in North Africa with a population of more than 100 million. Assiut University and its affiliated hospitals (Figure 1) are the largest academic tertiary care complex in Upper (southern) Egypt, serving more than 20 million people. Our setting is the Women's Health Hospital, Assiut University OBGYN specialized hospital with a capacity of 400 beds, 32 intermediate and 16 ICU beds. Numerous barriers face patients with gynecologic cancer in our region including soaring poverty levels, high cancer rates, and limited resources. There are no gynecologic oncology training programs in Egypt, so the care of patients with gynecologic cancer has overlapped between general gynecologists and surgical oncologists.

A journey of 1000 miles begins with a single step. Our journey started 10 years ago to develop a dedicated service for patients with gynecologic cancers. We developed the UTOPIA model (Uncover Travel Observe Plan Implement Aid). The goal of the model was that a fellow would travel to a specialized cancer center around the world, gain clinical and research knowledge, then return with a plan to implement that gained experience and disseminate it. In 2010 Dr Hisham Abou-Taleb traveled to Kyoto University, Japan. The following year Dr Ali Hamed traveled to Indiana University, USA. In 2013 Dr Ahmed Abdelbadee Hefnawy traveled to the Christie Cancer Center in the UK and then to Case Western Reserve University, USA. Following their return, they invited young aspiring clinicians from the gynecology, pathology, clinical oncology, radiology, and other specialties who shared the vision to make a difference in the lives of patients with gynecologic cancer through a multidisciplinary approach. The novel model at our setting even attracted physicians from the neighboring South Egypt Cancer Institute to join our team. This was the first regular gynecologic oncology multidisciplinary tumor board in Upper Egypt (Figure 2).

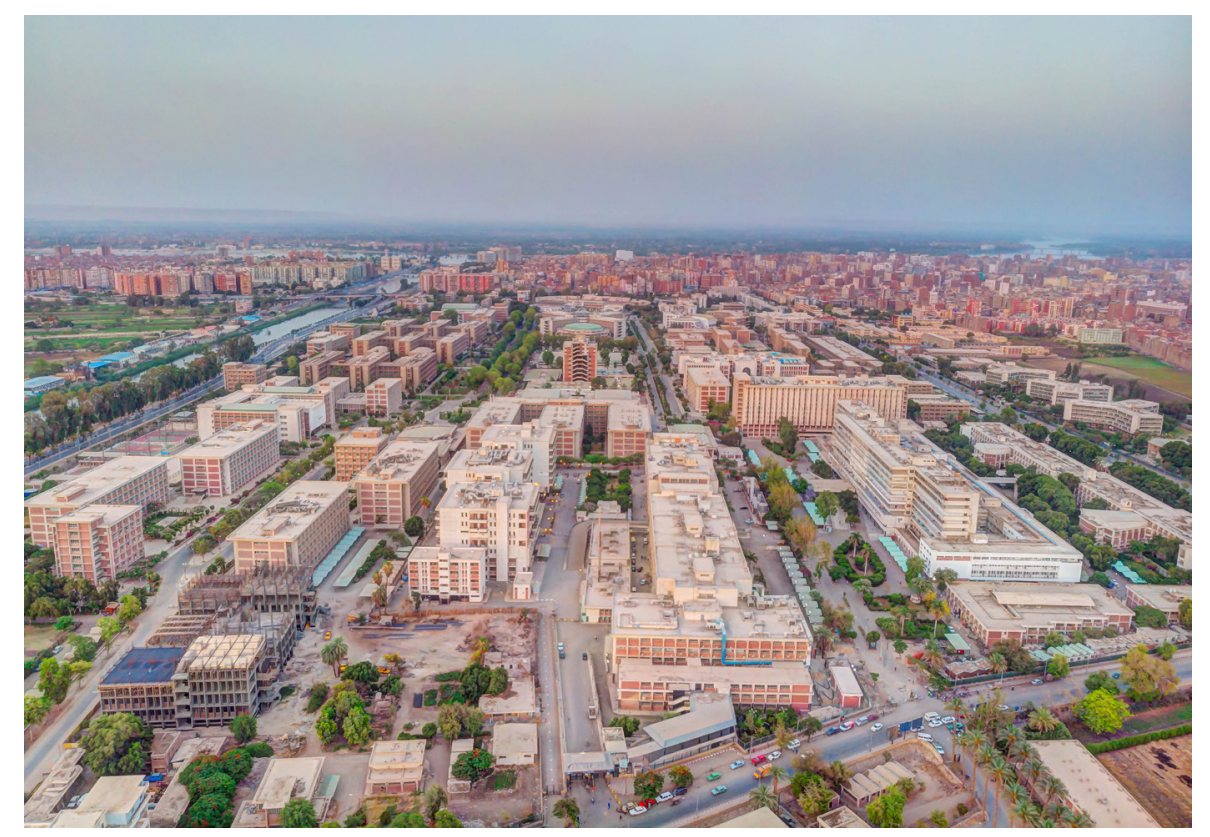

Figure 1 Overview of Assiut University Hospitals (courtesy of Mr Haytham Alhefnawy).

Our team combined the American, European, and Japanese experiences and guidelines and adapted them to create protocols guiding gynecologic cancer diagnosis and

management at our institution. Our model employed better organization of our limited resources. We created fast-track pathways for suspected gynecologic cancer

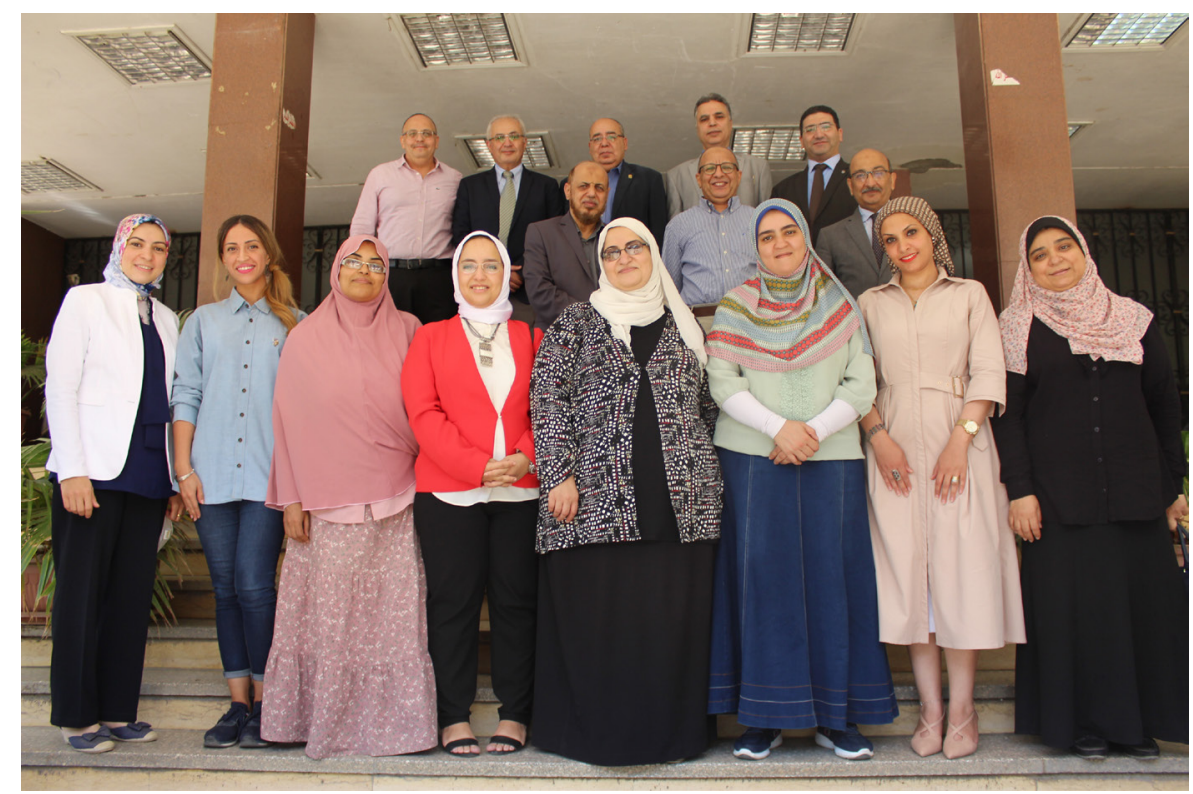

Figure 2 Assiut University gynecologic oncology multidisciplinary team. 

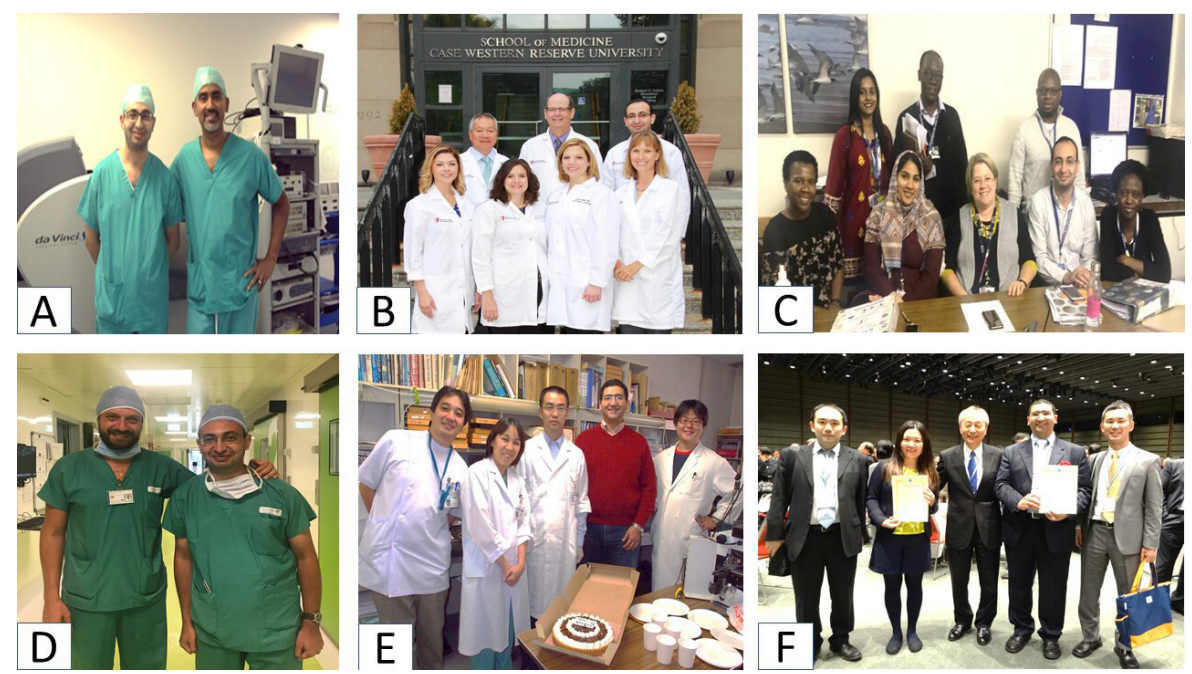

Figure 3 UTOPIA model application. (A) Dr Ahmed (left) at the Christie Cancer Center, Manchester, UK. (B) Dr Ahmed (second row, right) at Case Western Reserve University, Cleveland, USA. (C) Dr Ahmed (second from right) at Groote Schuur Hospital, Cape Town, South Africa. (D) Dr Ahmed (right) at Polyclinico Gemelli Hospital, Rome, Italy. (E) Dr Hisham (second from right) at Kyoto University, Japan. (F) Dr Hisham (second from right) receiving Best Gynecologic Oncology Research Award at the Japan Obstetrics and Gynecology Society 66th Annual Congress, Tokyo, Japan.

diagnoses. Surgical care was optimized to minimize complications. Challenging surgeries were discussed pre-operatively with our anesthesia team, thus performing radical surgeries and lymphadenectomies safely. Our general surgery team assisted in colorectal resection and hepatobiliary dissection procedures. Our vascular and reconstruction teams were consulted in complex surgery planning whenever vascular injuries were anticipated or flaps needed for reconstruction. ${ }^{1}$ Cases involving the urinary tract were discussed jointly with the urologic oncology team. While we lacked brachytherapy at our university, external beam radiation and conventional chemotherapeutic drugs were available.

Once our main service was well established, we explored further obstacles facing us such as the lack of funds, absence of palliative care, and insufficient access to minimally invasive surgery. Our team's vision to improve our services employed the UTOPIA model (Figure 3). In 2018, the expertise of the University of Cape Town, South Africa in palliative care helped extend our multidisciplinary team to include pain management, physical therapy, psychiatry, and clinical nursing staff. ${ }^{2}$ The following year we observed the skills of the surgeons in the gynecologic oncology division, University of Sacred Heart, Italy to introduce sentinel lymph node mapping in our surgeries. In 2020, with the support of the Union of International Cancer Control (UICC), we benefited from the expertise of the King Hussein Cancer Center, Jordan in providing comprehensive cancer care at an affordable cost. ${ }^{3}$ Our future vision is to develop the next generation of gynecologic oncologists through creation of a sub-specialized fellowship. We hope this generation will continue our journey to travel to corners of the world, collaborate internationally, and bring innovative experiences to strengthen our healthcare system and provide quality services to our patients.

Correspondence to Dr Ahmed Abdelbadee, Obstetrics \& Gynecology, Assiut University Faculty of Medicine, Assiut 71515, Egypt; ahmedhefnawy2015@gmail. com

Twitter Ahmed Abdelbadee @AhmedAHefnawy and Hisham Abou-Taleb @AbouTalebHisham

Acknowledgements The authors wish to acknowledge contributions from all the medical, surgical, and paramedical teams of Assiut University Hospitals for their continuous work to improve the standard of care given to our patients. Many respects to the late Professor Hossam Salem who led our team during this journey.

Collaborators From Assiut University Hospitals: Ihab El-Nashar, Elwany Elsnonsy, Ali H Hamed, Mohamed Khalaf, Ali Hussein, Etemad H Yasin, Abeer Refaiy, Dalia M Badary, Moemen M Hafez, Mahmoud F Sherif, Hisham Imam, Gehan Seifeldein, Heba Sabet, Radwa Abo El-kasem, Alshimaa Alsayed, Sara Hassanein, Sayed Hassaneen, Samir Shehata, Samy M Elgizawy, Tareq Salah, Reham Abdel-Wahab, Rehab F Mohamed, Rania Mahran, Summar Elmorshidy, Asmaa A Abdeltawab, Nada H Salah, Mariam Khalil, Safaa Kamel, Aziza Ferghali. From South Egypt Cancer Institute: Shimaa A Ahmed, Noha A Abdelmalik, Ahmed M Hefni.

Contributors All the authors have contributed to the writing and final approval of the manuscript. Our collaborators provided care to our patients through our multidisciplinary team.

Funding The authors have not declared a specific grant for this research from any funding agency in the public, commercial or not-for-profit sectors.

\section{Competing interests None declared.}

Patient consent for publication Not required.

Provenance and peer review Commissioned; internally peer reviewed.

C IGCS and ESGO 2021. No commercial re-use. See rights and permissions. Published by BMJ.

\section{Check for updates}

To cite Abdelbadee A, Abou-Taleb H. Int J Gynecol Cancer 2021;31:789-790.

Accepted 15 0ctober 2020

Published Online First 28 0ctober 2020

Int J Gynecol Cancer 2021;31:789-790.

doi:10.1136/ijgc-2020-002144

\section{ORCID iD}

Ahmed Abdelbadee http://orcid.org/0000-0003-44145247

\section{REFERENCES}

1 Abdelbadee A, Elnaggar A, Morsy M, et al. EP1147 Limb preserving surgery for necrotic vulvar cancer groin recurrence infiltrating the femoral artery and pubic bone. Int $J$ Gynecol Cancer 2019;29:A596-7.

2 Abdelbadee A, Abou-Taleb H, Abbas A, et al. Road map to setting up a palliative care service in a tertiary center gynecologic oncology unit in Egypt. J Glob Oncol 2018;4:176s

3 Hefnawy A. UICC blog: comprehensive cancer care in the Middle East, 2020. Available: https://www.uicc.org/blog/ comprehensive-cancer-care-middle-east 